glans ; one case of tertiary ulceration involving the whole penis and part of the scrotum ; one case of slow phagedænic ulcers of the prepuce and thighs; a case of very rapid sloughing phagedæna of chancre and bubo; and a case of gangrene of nearly the whole body of the penis.

As the limits of this paper will not allow of my giving the cases in detail, I shall only briefly notice their leading features. The shortest period during which the bath was made use of was one day; the longest was twelve days. Most of the twenty-two comparatively recent cases of phagedæena or sloughing attacking a chancre, or the initial lesion of syphilis, became healthy in from two to six days, and a few slight ones became so after one day. The most severe case was that of a strong healthy-looking man, aged twentyone, in whom a local sore on the penis and a bubo in the left groin were attacked by sloughing phagedæna, which extended so rapidly that in a few days the cavity in the groin had the diameter of one's fist. Here the sloughing ceased to extend after four days, but the bath was continued for eight days afterwards. Both chancre and bubo then rapidly healed. The case in which nearly the whole of the penis was lost was that of a man of broken-down constitution and drunken habits, aged fifty-seven. No satisfactory history could be obtained beyond the fact that a sore had appeared about two days after connexion, a month before admission into the hospital, by which time about two-thirds of the penis had sloughed. There was no extension after one day in the bath, and after four days the whole of the slough came away. In three weeks from the time of admission the patient went out with a stump about an inch long and nearly healed. There were no signs of syphilis.

In three $\operatorname{cases}^{7}$ morbid action reappeared, the ulcers having been healthy when the bath was discontinued. Perhaps in these it was left off too soon, or continuous immersion day and night might have succeeded. However, one of them got quite well eventually under other treatment. One was lost sight of after attending for some time as an out-patient. In the third the parts were quite healthy and healing when he was discharged, after having been three months in the hospital.

In three instances failure resulted. The first of these was a case of malignant syphilis with a very large sloughing primary sore, the patient being at the same time almost covered with an ulcerating syphilide. After nine days of the bath he refused to continue it. In the second case failure was due to the man being too stout to sit comfortably in the bath. The third and most complete failure was in a man who had suffered very severely from tertiary syphilis, and in whom the whole of the penis and part of the scrotum were involved in one large unhealthy ulceration. The pain was intense, and the patient said the bath aggravated it. The parts, after some time, healed entirely under large doses of iodide of potassium and opium and the local application of thick layers of cotton wool. This is the only case I have seen where pain was not quickly relieved by immersion. Mr. Coulson ${ }^{8}$ has recorded an instance in which the bath failed, although continued for eight successive days and nights, as well as during thirteen days, the patient being allowed to go to bed during the night. Mr. Hutchinson ${ }^{9}$ also mentions an unsuccessful case.

In order to obtain the full benefit from immersion it is necessary to bear in mind a few practical points. First, care must be taken to see that the affected part is thoroughly and constantly submerged; the penis has a tendency to float, and if the supply of water be too scanty, and the extremity of the organ be the seat of the ulcer, this may be altogether above the surface, and thus failure will occur. It is also imperative that the whole of the unhealthy part be exposed to the action of the water; if phimosis prevent this the foreskin should either be slit up or removed altogether. When the prepuce is simply divided the edges of the wound always swell more or less, and sometimes so much that the glans becomes almost buried, the discharge does not get freely away, and the length of time necessary for immersion is prolonged ; besides, a subsequent operation to remedy the deformity is generally necessary. Two lateral incisions, as recommended by Dr. R. W. Taylor, ${ }^{10}$ answer very well in completely exposing the parts, but here, also, a

7 All these were examples of the slowly-spreading non-inflammatory form of phagedæna. In none of the acute cases was there ever any relapse after the sore had once become healthy.

8 A Treatise on Syphilis, p. 145.

10 American Journal of Syphilography and Dermatology, 1872, p. 308. second operation is required for the removal of the flaps. Circumcision gives the best results, and was practised in several of the above cases, and as the wound, under immersion, scarcely ever takes on the diseased action, it may usually be done without fear. In case of troublesome hæmorrhage, either after operation or otherwise, solution of persulphate of iron may be applied, a bandage put on, and the bath deferred until the following day. Protection of prominent parts of the patient's body should not be forgotten. A few folds of an old blanket for him to sit on, and the same behind his back, answer very well in a hospital ; but in private practice air- or water-cushions may be used. Dr. Simmons recommends a large sponge for the patient to sit on. The bath should invariably be continued for at least a day after the ulcerated surface has become quite healthy, though it may often be continued with excel. lent effect until the sore has nearly or quite healed. Lastly, whenever the bath, limited to the daytime, fails, continuous immersion, as advised by Mr. Hutchinson, should always be resorted to.

Some of the advantages of immersion are : its effect in nearly always quickly relieving, and often in removing entirely, the severe pain attending phagedæna. Caustics are very rarely required. The ulcer is kept clean and free from discharge without pain or trouble. When a wound has to be made by the surgeon, its edges hardly ever become inoculated. The pain caused by frequent change of dressing is altogether avoided. The materials necessary for im. mersion being only those usually to be found in every house, it can be equally well carried out in private as in hospital practice.

In conclusion, I think it may be said that in the majority of cases of unhealthy ulceration and sloughing, whether local or syphilitic, immersion is the best and most speedy, as it is certainly the least painful, of the modes of treatment hitherto adopted.

Welbeck-street.

\section{A NEW SUSPENSORY BANDAGE.}

\section{BY CHAS. B. KEETLEY, F.R.C.S.,} ASSISTANT-SURGEON TO THE WEST LONDON HOSPITAL.

THE common suspensory bandage has these disadvantages : (1) if it is not fitted with a perineal band, the genital organs are pulled so far forwards as to cause an unsightly prominence beneath the trowsers; (2) if it has the ordinary perineal band, the anus gets chafed; (3) the tape in the hem round the margin of the bag chafes and cuts; (4) the perforations in the material of the bag are too fine for ventilation, consequently the bag becomes soaked with perspiration; (5) the hole for the penis is unnecessary, and only serves to let one testicle occasionally herniate through it.

The bandage $I$ wish to bring under notice is contrived to obviate the above objections. A median perineal band holds the genitalia well back. An india-rubber ring in this band prevents it from chafing the anus, and the anus from soiling it. Another india-rubber ring takes the place of the piece of tape above referred to. The bag is hand-made, knitted, and very open in its network; and the penis is simply allowed to hang over the front of the bag. The contrivance has been freely tried in cases of gonorrhcea, orchitis, varicocele, relaxed condition of the genitalia attended by hypochondriasis, and in other affections of those parts, and found to answer perfectly.

Messrs. Arnold and Sons, of West Smithfield, make the appliance in three sizes, at the price of the old bandage.

University College, London.-The Council of this College have awarded to Mr. H. F. Stokes, M.R.C.S., of Highbury (late house-surgeon of the hospital) the Atkinson-Morley Scholarship, of the value of $f 45$ per annum, tenable for three years, for special proficiency in surgery.

Stemvens's Hospital, Dublin. - At the close of the winter session the following prizes were awarded :Gold medals : Senior class, John Neill; middle class, William J. Trotter; junior class, Samuel R. Wills. Honorary certificates: Messrs. Blackham, Cruice, Cuthbert, Deckrell, Lane, Lawless, and Wilson. 